\title{
A Superspace Formulation for the Master Equation
}

\author{
Everton M. C. Abreu ${ }^{1,2}$ and Nelson R. F. Braga ${ }^{1}$ \\ ${ }^{1}$ Instituto de Física, Universidade Federal do Rio de Janeiro, \\ Caixa Postal 68528, 21945 Rio de Janeiro, RJ, Brazil \\ ${ }^{2}$ Instituto de Física, Universidade do Estado do Rio de Janeiro, \\ Rua Sao Francisco Xavier 524,CEP 20550 Rio de Janeiro, RJ, Brazil
}

\begin{abstract}
It is shown that the quantum master equation of the Field Antifield quantization method at one loop order can be translated into the requirement of a superfield structure for the action. The Pauli Villars regularization is implemented in this BRST superspace and the case of anomalous gauge theories is investigated. The quantum action, including Wess Zumino terms, shows up as one of the components of a superfield that includes the BRST anomalies in the other component. The example of W2 quantum gravity is also discussed.
\end{abstract}

PACS: $11.15,03.70$ 


\section{Introduction}

The gauge invariance principle is one of the basic ingredients in the search for a description of the fundamental processes involving elementary particles. Gauge invariance is translated at the quantum level into the fermionic rigid BRST invariance[1] and is important in the proof of unitarity and renormalizability of field theories [2].

The path integral quantization of gauge field theories poses some interesting problems. The naive integration over all the field configurations would lead to an over counting of physically equivalent ones. A mechanism of factoring out this over counting, at least for some special kind of gauge field theories was proposed by Faddeev and Poppov [3].

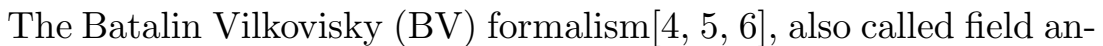
tifield quantization, is a Lagrangian BRST procedure that generalizes the Faddeev Poppov mechanism and also incorporates the idea 2] of including sources of the BRST transformations as independent variables as an important tool for deriving the Ward identities. The formalism is defined in an extended space that includes the fields and also the BRST sources, called antifields. In this space, the Ward identities, representing the BRST invariance of the vacuum functional, can be cast into a general expression, called master equation. One of the main goals of this general approach is that for the case of reducible gauge theories it furnishes a systematic way of building up the non trivial ghost for ghost structure. Also quantum corrections from the path integral measure, for anomalous gauge theories, can be calculated as long as a regularization procedure is introduced[7].

It is known that superspace formulations for gauge field theories can be build up in such a way that the BRST transformations are realized as translations in a Grasmannian coordinate[8]. It has also recently been shown [9] that the BV formalism at classical level (zero order in $\hbar$ ) can also be cast in such a BRST superspace form.

If we use the standard BV formulation, when we go to the BRST superspace we will in general find constrained superfields. As will be discussed in section $\mathbf{2}$, functional differentiation with respect to superfields will be essential in finding a superspace version for the operator $\Delta$, but for constrained superfields we can not find a general definition for functional derivatives. In order to overcome this obstacle we will consider an alternative derivation for the BV action proposed 
in $\mid 10]$. In this so called collective approach to BV, the set of fields of the classical theory and also the ghosts, antighosts and auxiliary fields associated to the original gauge symmetries is trivially doubled. This leads to new (trivial) shift symmetries. These extra symmetries can then be gauge fixed (adding new ghosts antighosts and auxiliary fields) in such a way that the BV action is recovered after the extra fields are integrated out. The antighosts of the trivial symmetries are identified as playing the role of the associated antifields. The transformations of the fields are chosen in such a way that at least for the superfields that will be relevant in building the superspace $\Delta$ operator the components will be independent.

In the present article we will investigate a superspace version of the field antifield formalism at one loop order in $\hbar$. We will find out that the master equation implies a certain structure for the superfield associated to the quantum action. We will also see that the Pauli Villars regularization can be formulated in this BRST superspace. A well defined meaning can thus be given to the superfield structure of the quantum action. The example of W2 gravity will nicely illustrate the formulation.

The article is organized as follows: in section $\mathbf{2}$ we discuss the superspace formulation for the BRST symmetry, explaining why do we use the collective field approach rather then the usual BV. In section 3 we review the superspace formulation for BV at classical level. In section 4 we present the general form of the master equation and of the superfield associated to the quantum action at one loop order. Section $\mathbf{5}$ is devoted to the Pauli Villars regularization in superspace. In section 6 we show the form of the superfield action at one loop order. The example of W2 gravity is discussed in section $\mathbf{7}$ and section 8 contains some concluding remarks.

\section{BRST superspace}

Superspace formulations for the BRST transformation 8] are obtained by associating to each field $\phi(x)$ a (BRST) superfield of the form:

$$
\Phi(x, \theta)=\phi(x)+\theta \delta \phi(x)
$$

where $\delta \phi(x)$ is the BRST transformation of $\phi(x)$. The BRST transformations are then realized as translations in the $\theta$ variable. 


$$
\delta \Phi(x, \theta)=\frac{\partial}{\partial \theta} \Phi(x, \theta)
$$

In order to apply this idea to the BV Master equation at one loop order, it is crucial that we define in a very precise way functional derivatives with respect to superfields. Let us start considering a general bosonic superfield of the form:

$$
\Lambda(x, \theta)=A(x)+\theta B(x)
$$

where $A(x)$ and $B(x)$ are independent quantities:

$$
\frac{\delta B(x)}{\delta A\left(x^{\prime}\right)}=0 ; \quad \frac{\delta A(x)}{\delta B\left(x^{\prime}\right)}=0
$$

If we define a functional derivative so as to satisfy:

$$
\frac{\delta \Lambda(x, \theta)}{\delta \Lambda\left(x^{\prime}, \theta^{\prime}\right)}=\delta\left(x-x^{\prime}\right) \delta\left(\theta^{\prime}-\theta\right)=\left(\delta\left(x-x^{\prime}\right)\right)\left(\theta^{\prime}-\theta\right)
$$

we recover, for superspace functionals, the usual interpretation of the functional derivative, as for example:

$$
\frac{\delta}{\delta \Lambda(x, \theta)} \int d x^{\prime} \int d \theta^{\prime}\left(\Lambda\left(x^{\prime}, \theta^{\prime}\right)\right)^{2}=2 \Lambda(x, \theta)
$$

it is important to remark that this superfield functional derivative has a Grassmanian parity opposite to the associated field. We can also express the derivative with respect to the superfield in terms of derivatives with respect to it's components:

$$
\frac{\delta}{\delta \Lambda(x, \theta)}=\frac{\delta}{\delta B(x)}+\theta \frac{\delta}{\delta A(x)}
$$

Considering now the BRST superfields of eq. (1), condition (4) of independent components clearly does not hold in general. Actually one loop level corrections are just associated to the contributions from (singular) terms like

$$
\frac{\delta\left(\delta_{B R S T} \phi\right)}{\delta \phi}
$$

we will thus be dealing in general with constrained superfields. 
A simple way to realize that for the BRST superfields of eq.(1) the naive application of superfield functional derivatives would lead to contradictory results is to consider the case of a field with vanishing BRST transformation, as for example the ghost fields in QED. For the associated superfield, with no $\theta$ component, if we try to define a functional derivative satisfying (5) we would arrive at the contradiction that the functional in (6) vanishes and would have a non vanishing derivative.

In standard supersymmetry a similar situation happens when one considers chiral or antichiral superfields. A functional derivative can be defined for these special constrained superfields but (5) is replaced by an appropriate version that takes the particular constraint into account. It is however impossible to define functional derivatives (and also path integration) for general constrained superfields [11].

In order to have a general superspace version for the BV master equation at one loop order we should find a superspace version of the operator $\Delta$, that involves two functional derivatives. As we have seen, just substituting fields by BRST superfields would be meaningless unless one finds a general definition for their functional derivatives. An interesting way to overcome this obstruction is to use the so called collective field approach to BV. As we will see in the following sections, the BRST algebra in this case will be such that, at least for the fields that will be used in the $\Delta$ operator, conditions (4) hold.

\section{Superspace Formulation at Classical Level}

In reference [9] a superspace formulation for the collective field approach to the Batalin Vilkovisky action at the order zero in $\hbar$ was presented for the case of the Yang Mills theory. Here we will briefly review this formulation, presenting it in a general way for gauge theories with closed gauge algebra.

Considering a gauge field theory characterized by a classical action $S_{0}\left[\phi^{i}\right]$ we introduce ghosts, antighosts and auxiliary fields associated to the original gauge invariance of $S_{0}$ in the usual way. The new enlarged set of fields is then denoted as $\phi^{A}$. These fields realize the BRST algebra represented as: 


$$
\delta_{0} \phi^{A}=R^{A}[\phi]
$$

Then we introduce a new set of fields called collective fields $\tilde{\phi}^{A}$ and replace everywhere $\phi^{A}$ by $\phi^{A}-\tilde{\phi}^{A}$. This way we double the field content of the theory and at the same time associate to each field a new trivial shift symmetry. In order to gauge fix these new symmetries we introduce new ghosts, antighosts and auxiliary fields, represented respectively as: $\pi^{A}, \phi^{*} A$ and $B^{A}$. We have a large freedom in choosing the BRST transformations for this enlarged set of fields. Following [10] let us define the enlarged BRST algebra as :

$$
\begin{aligned}
\delta \phi^{A} & =\pi^{A} \\
\delta \tilde{\phi}^{A} & =\pi^{A}-R^{A}[\phi-\tilde{\phi}] \\
\delta \pi^{A} & =0 \\
\delta \phi^{* A} & =B^{A} \\
\delta B^{A} & =0
\end{aligned}
$$

and the total action as:

$$
S_{\text {col. }}=S_{0}\left[\phi^{i}-\tilde{\phi}^{i}\right]-\delta\left(\phi^{* A} \tilde{\phi}^{A}\right)+\delta \psi\left[\phi^{A}\right]
$$

where $\psi\left[\phi^{A}\right]$ is a fermionic functional representing the gauge fixing of the original symmetries (9). The BV gauge fixed classical action is obtained if one functionally integrates the vacuum functional associated with $S$ over $\pi^{A}, \tilde{\phi}^{A}$ and $B^{A}$.

Now, the BRST superspace formulation is obtained introducing the superfields

$$
\begin{aligned}
\Phi^{A}(x, \theta) & =\phi^{A}(x)+\theta \pi^{A}(x) \\
\tilde{\Phi}^{A}(x, \theta) & =\tilde{\phi}^{A}(x)+\theta\left(\pi^{A}(x)-R^{A}[\phi-\tilde{\phi}]\right) \\
\Phi^{* A}(x, \theta) & =\phi^{* A}(x)+\theta B^{A}(x)
\end{aligned}
$$

We can also associate superfields to the ghosts and the auxiliary fields of the shift symmetry but they would have a trivial structure: $\underline{\Pi}^{A}(x, \theta)=\pi^{A}(x), \underline{B}^{A}(x, \theta)=B^{A}(x)$.

Considering the set (12) we can define a superfield action as: 


$$
\underline{S}_{c o l .}=S_{0}\left[\Phi^{i}-\tilde{\Phi}^{i}\right]-\frac{\partial}{\partial \theta}\left\{\Phi^{* A} \tilde{\Phi}^{A}+\psi\left[\Phi^{a}\right]\right\}
$$

This object actually has a trivial superspace structure as it's $\theta$ component is zero $\left(\underline{S}_{c o l}=S_{\text {col. }}\right.$. ). It may thus seem meaningless at this stage to associate a superfield to the action. We will see however in the next section that when higher order terms in $\hbar$ are taken into account the situation is rather different. At Classical level, $S_{\text {col. }}$ is BRST invariant, therefore the associated superfield must have a zero $\theta$ component, expressing what we will see in the next section to correspond to the zero order term of the master equation. We will see in the next section that at higher in $\hbar$ the quantum action is not BRST invariant and the associated superfield structure will not be trivial.

Concluding this section we remark that in the collective field approach of [10], presented here, the fields $\phi^{* A}$ that play the role of antifields are substituted by the gauge fixing conditions, after integration over the auxiliary fields. In other words, we get the BV gauge fixed action. One may however be interested in an action that still involves the antifields as for example if one wants to build up an effective action in terms of classical fields and antifields [5]. In order to show that the collective field approach can also reproduce this non completely gauge fixed result we can add to $\underline{S}_{c o l}$. the term:

$$
\frac{\partial}{\partial \theta}\left(\Phi^{A} \underline{\varphi}^{A}\right)=\varphi^{A} \pi^{A}
$$

where $\varphi^{A}$ are BRST invariant external fields with parity opposite to that of $\phi^{A}$. Integration over the auxiliary fields would recover the BV action with external antifields as:

$$
\begin{gathered}
\exp \left\{\frac{i}{\hbar} S_{B V}\left[\phi^{A}, \frac{\partial \psi}{\partial \phi^{A}}+\varphi^{A}\right]\right\}= \\
=\int D \tilde{\phi}^{A} D \phi^{*} D \pi^{A} D B^{A} \exp \left\{\underline{S}_{c o l .}+\frac{\partial}{\partial \theta} \Phi^{A} \underline{\varphi}^{A}\right\}
\end{gathered}
$$

for $\varphi^{A}=0$ this reduces to the gauge fixed BV action. For $\psi=0$ we get the non gauge fixed action. 


\section{Superspace version of the Master equa- tion}

We will first investigate the BRST variation of the quantum action in the standard field antifield quantization method. Then we will see the corresponding behavior in the collective field approach. We will consider the case of gauge theories with closed gauge algebra. Anomalies may in general have a non trivial dependence on the antifields [13, 14]. We will however consider here a regularization procedure that will only provide for the antifield independent part of $\Delta S$. For gauge theories with closed algebras one can consistently consider this part separately[15]. Recently proposed non-local[16] or antifield dependent 17 regularization procedures are also out the scope of our present superspace formulation.

The condition of gauge independence of the vacuum functional

$$
Z_{\Psi}=\int \prod D \Phi^{A} \exp \left(\frac{i}{\hbar} W\left(\phi^{A}, \phi^{* A}=\frac{\partial \psi}{\partial \phi^{A}}\right)\right.
$$

is translated into the so called (quantum) master equation:

$$
\frac{1}{2}(W, W)=i \hbar \Delta W
$$

where the antibracket is defined as: $(X, Y)=\frac{\partial_{r} X}{\partial \phi^{a}} \frac{\partial_{l} Y}{\partial \phi^{* a}}-\frac{\partial_{r} X}{\partial \phi^{* a}} \frac{\partial_{l} Y}{\partial \phi^{a}}$ and the operator Delta as: $\Delta \equiv \frac{\partial_{r}}{\partial \phi^{a}} \frac{\partial_{l}}{\partial \phi_{a}^{*}}$

The quantum action can be expanded in a power series in $\hbar$ as: $W\left(\phi^{A}, \phi^{* A}\right)=S\left(\phi^{A}, \phi^{* A}\right)+\sum_{p=1}^{\infty} \hbar^{p} M_{p}\left(\phi^{A}, \phi^{* A}\right)$ we will be concerned here just with the first two terms, since we are considering just one loop corrections. In order to investigate the behavior of $W$ with respect to BRST transformations, let us consider the BRST transformation for some quantity $\mathrm{X}$ in the standard BV language [12]:

$$
\delta X=(X, W)-i \hbar \Delta X
$$

if we choose $X=W$ and consider that we are dealing with a non anomalous theory such that the master equation is satisfied we get from $(17)$ and (16):

$$
\delta W=i \hbar \Delta W
$$

This condition is equivalent to the master equation. 
Under our present assumption that $M_{1}$ does not depend on the antifields we have (up to one loop order only)

$$
\begin{aligned}
\delta S & =0 \\
\delta M_{1} & =i \Delta S
\end{aligned}
$$

Going now to the collective field case. We see from (13) that the extended action $S_{c o l}$. is also BRST invariant. For the action of the $\Delta$ operator we get a similar result in the collective field approach and in the standard one:

$$
\Delta S=\Delta S_{\text {col. }}=\frac{\partial_{r}}{\partial \phi^{A}} \frac{\partial_{l}}{\partial \phi^{* A}} S_{c o l}
$$

it should be noted, however that in the collective field case $\phi^{* A}$ are not antifields but rather antighosts of the shift symmetry. Therefore, at one loop order, we must build up a superfield

$$
\underline{M}_{1}\left[\Phi^{A}-\tilde{\Phi}^{A}\right]=M_{1}\left[\phi^{A}-\tilde{\phi}^{A}\right]+\theta i \Delta S_{c o l} .
$$

and the general form of the superfield action will be:

$$
\underline{W}=W+\theta i \hbar \Delta W
$$

Actually this expression for the superfield $\underline{W}$ is just formal, in the same way as the master equation (16) itself. We can only have a precise interpretation for terms involving the operator $\Delta$, where two functional derivatives act on the same space time point if some regularization procedure is applied [7]. We will show in the next section how the Pauli Villars regularization procedure can be implemented in this superspace.

Let us now define, in the collective field space, the operator

$$
\underline{\Delta} \equiv \int d x \int d \theta \int d \theta^{\prime} \frac{\delta_{r}}{\delta \Phi^{A}(x, \theta)} \frac{\delta_{l}}{\delta \Phi^{* A}\left(x, \theta^{\prime}\right)}
$$

where we have indicated explicitly the integrations over space-time and Grassman variables, omitted in the previous expressions, because of the non trivial form (the functional derivatives are taken in the same space time point but in different Grassman coordinates). 
Looking at (12) we see that the superfields involved in $\underline{\Delta}$ satisfy (㺼). Therefore the functional derivatives are well defined and we can also use the decomposition in components (7) in order to calculate:

$$
\underline{\Delta} \underline{S}_{c o l .}=\underline{\Delta} \int d x \int d \theta\left(-\Phi^{* A}(x, \theta) \tilde{\Phi}^{A}(x, \theta)\right)=\int d x \frac{\delta_{r}\left(R^{A}(x)\right)}{\delta \phi^{A}(x)}
$$

that is precisely the result that one obtains in the standard BV formalism if the $\Delta$ operator is naively applied to the Classical Action. We have thus found a superspace representation for this operator.

The master equation in superspace then reads:

$$
\frac{\partial}{\partial \theta} \underline{W}=i \hbar \underline{\underline{\Delta}}
$$

or order by order:

$$
\frac{\partial}{\partial \theta} \underline{S}=0 ; \quad \frac{\partial}{\partial \theta} \underline{M}_{1}=i \underline{\Delta S}
$$

At this point one could question about the lack of an antibraket structure in the present superspace approach. However, looking at (2) and (25) one realizes that the role of generator of BRST transformations is essentially played by the differentiation with respect to $\theta$. Therefore, enlarging the configuration space with the variable $\theta$, we are equipping it with Grassmanian translations that reproduce the effect of the antibrackets. So, this structure is not necessary and would be redundant.

\section{One loop order regularization in su- perspace}

The Pauli Villars regularization procedure is the most suitable for the BV formalism at one loop order [6, 7, 18, 19]. We will consider, for simplicity, the case of just one Pauli Villars (PV) field associated to each field of the theory. In some cases one needs a set of PV fields but this modification would not change the superspace structure, as it will be seen in the example. In the present superspace formulation the field content of the theory is enlarged by the addition of the collective fields and the gauge fixing structure of the associated shift symmetries. 
We will build up a Pauli Villars superfield action corresponding to a collective field version of the standard PV action, or equivalently, to a PV partner of action $\underline{S}_{\text {col. }}$ of eq. (13):

$$
\begin{aligned}
\underline{S}_{P V} & =\frac{1}{2}\left(\underline{\chi}^{A}-\underline{\tilde{\chi}}^{A}\right)(T O)_{A B}\left(\underline{\chi}^{B}-\underline{\tilde{\chi}}^{B}\right) \\
& -\frac{1}{2} M\left(\underline{\chi}^{A}-\underline{\tilde{\chi}}^{A}\right) T_{A B}\left(\underline{\chi}^{B}-\underline{\tilde{\chi}}^{B}\right)-\frac{\partial}{\partial \theta}\left(\underline{\chi}^{* A} \underline{\tilde{\chi}}^{A}\right)
\end{aligned}
$$

as in [7], the matrix $T$ is an arbitrary invertible one while $T O$ is :

$$
(T O)_{A B}=\frac{\partial_{l}}{\partial \Phi^{A}} \frac{\partial_{l}}{\partial \Phi^{B}} S^{\prime}\left(\Phi^{A}, \frac{\partial \psi}{\partial \phi^{A}}\right)
$$

where $S^{\prime}\left(\Phi^{A}, \frac{\partial \psi}{\partial \phi^{A}}\right)$ is obtained from the original action (11) after removing the collective fields

$$
\left.\exp \left(\frac{i}{\hbar} S^{\prime}\left(\Phi^{A}, \frac{\partial \psi}{\partial \phi^{A}}\right)\right)\right)=\int D \tilde{\phi}^{A} D \pi^{A} D B^{A} \exp \left(\frac{i}{\hbar} \underline{S}_{c o l .}\right)
$$

In order to build up the PV superfields we must define their enlarged BRST algebra. We define the matrix

$$
K_{A B}=\frac{\partial_{l}}{\partial \Phi^{* A}} \frac{\partial_{r}}{\partial \Phi^{B}} \underline{S}_{c o l}
$$

where $\underline{S}$ is the action of eq. (13), that actually has no $\theta$ component, and impose that the non extended (without collective fields) BRST algebra for the PV fields reads:

$$
\delta_{0} \chi^{A}=K_{A B} \chi^{B}
$$

Following the steps of section $\mathbf{2}$ we find the enlarged algebra for the PV fields and build up the associated superfields:

$$
\begin{aligned}
\underline{\chi}^{A}(x, \theta) & =\chi^{A}(x)+\theta \pi^{[\chi] A} \\
\underline{\tilde{\chi}}^{A}(x, \theta) & =\tilde{\chi}^{A}(x)+\theta\left(\pi^{[\chi] A}-K_{A B}\left(\chi^{B}-\tilde{\chi}^{B}\right)\right) \\
\underline{\chi}^{* A}(x, \theta) & =\chi^{* A}(x)+\theta B^{[\chi] A}
\end{aligned}
$$

As usual, the PV fields are defined formally in such a way that their one loop contributions have a minus sign relative to the original 
fields. The action of the operator $\underline{\Delta}$ on the regularized total action is thus:

$$
\begin{aligned}
\underline{\Delta}\left(\underline{S}+\underline{S}_{P V}\right) & \equiv \int d x \int d \theta \int d \theta^{\prime}\left(\frac{\delta_{r}}{\delta \Phi^{A}(x, \theta)} \frac{\delta_{l}}{\delta \Phi^{* A}\left(x, \theta^{\prime}\right)}\right. \\
& \left.+\frac{\delta_{r}}{\delta \underline{\chi}^{A}(x, \theta)} \frac{\delta_{l}}{\delta \underline{\chi}^{* A}\left(x, \theta^{\prime}\right)}\right)\left(\underline{S}+\underline{S}_{P V}\right)=0
\end{aligned}
$$

The regularized form of $\Delta S$ in the non superspace case shows up in the violation of the zero order master equation associated to the presence of the mass term. In superspace this absence of BRST invariance of the total (regularized) classical action $S_{T}=S+S_{P V}$ is translated into the presence of a $\theta$ component in the corresponding superfield:

$$
\underline{S}_{T}=\underline{S}+\underline{S}_{P V}=S_{T}+\theta \delta S_{T}
$$

The general form of $\delta S_{T}$ is

$\delta S_{T}=M\left(\left(\chi^{A}-\tilde{\chi}^{A}\right) T_{A C} K_{B}^{C}\left(\chi^{B}-\tilde{\chi}^{B}\right)+\frac{1}{2}\left(\chi^{A}-\tilde{\chi}^{A}\right) \delta T_{A B}\left(\chi^{B}-\tilde{\chi}^{B}\right)\right)$

Integration over the fields $\pi^{[\chi] A}, B^{[\chi] A}$ and $\tilde{\chi}^{A}$ removes the extended collective field structure, recovering the usual result as in [7], that corresponds in (35) just to the absence of the collective tilde fields. The next step would be to integrate over the PV fields. We will not repeat this procedure here as it is widely discussed in the literature 6, 7, 15, 18, 19. Let us assume that a regularized form of the BRST

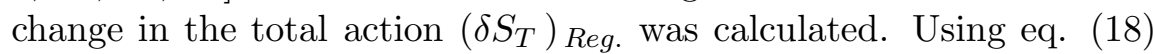
up to one loop order terms we find the relation between the BRST variation of the regularized action and the desired regularized $\Delta S$ :

$$
i \hbar(\Delta S)_{R e g}=\left(\delta S_{T}\right)_{R e g}
$$

\section{Anomalous gauge theories}

Genuine anomalies are characterized by a violation of the master equation [7]. For this kind of theories, it is not possible to find an $M_{1}$ term in the original space of fields and antifields, such that the master 
equation (16) is satisfied. As already explained, in the present superspace formulation we are considering the particular case in which the regularization procedure provides just the antifield independent contributions to $\Delta S$. We will thus consider only antifield independent anomalies and Wess Zumino terms. Under this assumption, the violation of the master equation is of the form:

$$
\Delta W+\frac{i}{2 \hbar}(W, W)=\mathbf{A}=c^{\alpha} A_{\alpha}
$$

The symmetries associated to the ghosts $c^{\alpha}$ are said to be broken by the anomalous behavior of the path integral measure. In this case the BRST transformation for the action has the form

$$
\delta W=i \hbar \Delta W-2 i \hbar c^{\alpha} A_{\alpha}
$$

The superfield associated to the quantum action will then look like

$$
\underline{W}=W+\theta\left(i \hbar \Delta W-2 i \hbar c^{\alpha} A_{\alpha}\right)
$$

It is interesting to discuss in our superspace formulation the mechanism of restoring gauge invariance by the inclusion of additional degrees of freedom associated to the (broken) gauge group, proposed by Faddeev and Shatashvili 20]. In the BV formalism this mechanism is implemented by enlarging the field antifield space 21 by including fields associated to the gauge group. This way one can find a description for a (potentially) anomalous gauge theory in which the classical symmetries are realized at the quantum level, at the cost of some of the gauge group degrees of freedom becoming dynamical. In the present one loop level superspace formalism this, so called Wess Zumino mechanism, corresponds to finding out a superfield (involving the additional field antifield pairs):

$$
\underline{M}_{1}=M_{1}+\theta(i \Delta S)_{\text {Reg }}
$$

such that the superfield action takes the non anomalous form (22) and one says that the anomalies have been canceled.

As remarked in [22], another interesting interpretation for this mechanism of canceling the anomalies in the original gauge symmetries, is that actually the anomalies are not canceled but shifted to a trivial sector of symmetries. One arrives at this result considering that the extra fields that realize the Wess Zumino mechanism are not 
present at the classical level and one should thus include in the classical action a gauge fixing term associated to the invariance with respect to any shift in these fields . Taking this point of view, the form of the superfield action, even after the Wess Zumino term is included, is still as in (37) the only difference is that the anomalies are shifted to extra ghosts $d^{\beta}$ associated to the (broken) trivial shift symmetries of the additional fields. This situation will be clarified in the example of the next section.

\section{Example}

Let us consider W2 gravity theory as an interesting example of an anomalous gauge theory that can be cast into the present superspace $\mathrm{BV}$ formulation. The classical theory is described by

$$
S_{0}=\frac{1}{2 \pi} \int d^{2} x\left[\partial \phi \bar{\partial} \phi-h(\partial \phi)^{2}\right]
$$

The BRST algebra associated to this theory is:

$$
\begin{aligned}
\delta_{0} \phi & =c \partial \phi \\
\delta_{0} h & =\bar{\partial} c-h \partial c+\partial h c \\
\delta_{0} c & =(\partial c) c
\end{aligned}
$$

where $c$ is the ghost associated with the original gauge invariance of $S_{0}$.

Now we follow the procedure of section $\mathbf{2}$ and enlarge the field content of the theory introducing the collective fields associated to $\phi, h$ and $c$, represented by tilde fields, the ghosts, antighosts and auxiliary fields. Then we build up the superfields $\Phi(x, \theta), \tilde{\Phi}(x, \theta), \Phi^{*}(x, \theta)$ , $H(x, \theta), \tilde{H}(x, \theta), H^{*}(x, \theta), \eta(x, \theta)=c(x)+\theta \delta c(x), \tilde{\eta}(x, \theta), \eta^{*}(x, \theta)$ as in $(12)$.

We will adopt the notation $\sigma^{\prime}=\sigma-\tilde{\sigma}$ for all fields and superfields in the rest of the section. The superfield action at classical level is:

$$
\underline{S}=\underline{S}_{0}+\underline{S}_{1}+\underline{S}_{2}
$$

with the collective field version of the classical action: 


$$
\underline{S}_{0}=\frac{1}{2 \pi} \int d^{2} x\left[\partial \Phi^{\prime} \bar{\partial} \Phi^{\prime}-H^{\prime}\left(\partial \Phi^{\prime}\right)^{2}\right]
$$

the gauge fixing of the shift symmetry:

$$
\underline{S}_{1}=-\frac{\partial}{\partial \theta} \int d^{2} x\left[\Phi^{*} \tilde{\Phi}+H^{*} \tilde{H}+\eta^{*} \tilde{\eta}\right]
$$

and the gauge fixing for the original symmetry:

$$
\underline{S}_{2}=\frac{\partial}{\partial \theta} \int d^{2} x \psi(\Phi, H, \eta)
$$

To realize the Wess Zumino mechanism one includes an extra field $\rho$ transforming according to the original gauge transformations associated to the ghost $c$ and also with an additional shift symmetry associated to an extra ghost $d$, representing the absence of this field at classical level [19]:

$$
\delta_{0} \rho=\partial c+c \partial \rho+d
$$

We introduce a collective field structure for this field, and associate to it the superfields $\Omega(x, \theta), \tilde{\Omega}(x, \theta), \Omega^{*}(x, \theta)$ as in (12). Usually one is interested in calculating the contributions from the matter fields $\phi$ only, considering the field $h$ as a background. Therefore we introduce, as in [22], a Pauli Villars field associated to $\phi$ that will be represented as $\chi$ and define the PV superfield action:

$$
\underline{S}_{P V}=\underline{S}_{P V 0}+\underline{S}_{P V 1}+\underline{S}_{M}
$$

with the first two terms analogous to the corresponding terms of the original fields:

$$
\begin{gathered}
\underline{S}_{P V 0}=\frac{1}{2 \pi} \int d^{2} x\left(\partial \underline{\chi}^{\prime} \bar{\partial} \underline{\chi}^{\prime}-H^{\prime}\left(\partial \underline{\chi}^{\prime}\right)^{2}\right) \\
\underline{S}_{P V 1}=-\frac{\partial}{\partial \theta} \int d^{2} x \underline{\chi}^{*} \underline{\tilde{z}}
\end{gathered}
$$

and the mass term:

$$
\underline{S}_{M}=-\frac{1}{2 \pi} M^{2} \int d^{2} x \underline{\chi}^{\prime 2} e^{\alpha \Omega^{\prime}}
$$

The PV superfields involved in this action are: 


$$
\begin{aligned}
\underline{\chi}(x, \theta) & =\chi(x)+\theta \pi^{[\chi]}(x) \\
\underline{\tilde{\chi}}(x, \theta) & =\tilde{\chi}(x)+\theta\left(\pi^{[\chi]}(x)-c^{\prime}(x) \partial \chi^{\prime}(x)\right) \\
\underline{\chi}^{*}(x, \theta) & =\chi^{*}(x)+\theta B^{[\chi]}(x)
\end{aligned}
$$

Defining now the total action as

$$
\underline{S}_{T}=\underline{S}+\underline{S}_{P V}=S_{T}+\theta \delta S_{T}
$$

we have:

$$
\delta S_{T}=\frac{1}{2 \pi} \int d^{2} x M^{2} \chi^{\prime 2}\left((1-\alpha) \partial c^{\prime}-\alpha d^{\prime}\right) e^{\rho^{\prime} \alpha}
$$

At this point we arrive at the standard (non superspace) results. Actually one needs a set of PV fields $\chi^{i}$ in order to regularize the above expression. They are, however, all of the same form and, in our superspace formulation, will all have actions like (41). The regularized result (after integrating out the PV fields) is 19, 22 :

$$
\begin{aligned}
& \frac{1}{\hbar}\left(i \delta S_{T}\right)_{R e g}=(\Delta S)_{R e g} \\
= & \frac{1}{12 \pi} \int d^{2} x\left[\left((1-\alpha) \partial c^{\prime}-\alpha d^{\prime}\right)\left(\partial^{2} h^{\prime}-\alpha\left(\partial \bar{\partial} \rho^{\prime}\right)-\partial\left(h^{\prime} \partial \rho^{\prime}\right)\right)\right]
\end{aligned}
$$

The superfield action at one loop order, using this regularization will then have the general form:

$$
\underline{M}_{1}(\alpha)=M_{1}(\alpha)+\theta\left(i(\Delta S)_{R e g}(\alpha)+\mathbf{A}(\alpha)\right)
$$

where $M_{1}(\alpha)$ is the Wess Zumino term. It is possible to choose this term in such a way that the anomaly is always shifted to the trivial symmetry associated to the ghost $d$. For the $\alpha=0$ case the appropriate choice is:

$$
\underline{M}_{1}(\alpha=0)=\frac{1}{12 \pi}\left\{\frac{1}{2} \partial \Omega^{\prime} \bar{\partial} \Omega^{\prime}-\frac{1}{2} H^{\prime}\left(\partial \Omega^{\prime}\right)^{2}+H^{\prime} \partial^{2} \Omega^{\prime}\right\}
$$




$$
\begin{aligned}
& =\frac{1}{12 \pi}\left\{-\frac{1}{2} \rho^{\prime} \partial \bar{\partial} \rho^{\prime}+\frac{1}{2} \rho^{\prime} \partial h^{\prime} \partial \rho^{\prime}+\frac{1}{2} \rho^{\prime} h^{\prime} \partial^{2} \rho^{\prime}+h^{\prime} \partial^{2} \rho^{\prime}\right\} \\
& +\theta \frac{1}{12 \pi}\left(-c^{\prime} \partial^{3} h^{\prime}+d^{\prime} \partial^{2} h^{\prime}-d^{\prime} \partial \bar{\partial} \rho^{\prime}-h^{\prime} \partial d^{\prime} \partial \rho^{\prime}\right) \\
& =M_{1}+\theta(i \Delta S+d \bar{A})
\end{aligned}
$$

with

$$
\bar{A}=\frac{1}{12 \pi}\left(\partial^{2} h^{\prime}-\partial \bar{\partial} \rho^{\prime}+\partial h^{\prime} \partial \rho^{\prime}+h^{\prime} \partial^{2} \rho^{\prime}\right)
$$

Considering now $\alpha=1$, it can be seen from (47) that in this case the regularization procedure itself leads to an anomaly only in the trivial symmetry associated to $d$. We can thus choose:

$$
\underline{M}_{1}(\alpha=1)=0
$$

and the anomaly will be:

$$
\begin{aligned}
\mathbf{A}(\alpha=1) & =-i(\Delta S)_{R e g}(\alpha=1) \\
& =\frac{1}{12 \pi} \int d^{2} x d^{\prime}\left(\partial^{2} h^{\prime}-\partial \bar{\partial} \rho^{\prime}+h^{\prime} \partial \rho^{\prime}\right)
\end{aligned}
$$

Integration over the auxiliary fields leads to the usual non superspace results, but it is important to stress that the superspace formulation requires the presence of the collective fields and the associated gauge fixing structure.

\section{Conclusion}

Although BRST superspace formulations for gauge theories have been known for a while [8], anomalous gauge theories have not yet been considered in this context. The Batalin Vilkovisky procedure represents a very powerful framework for the quantization of this kind of theories. We have shown that the (formal) master equation of the BV formalism can be represented as the requirement of a (formal) superspace structure for the quantum action. At one loop order, using the collective field approach to BV, we have shown that the Pauli Villars regularization procedure can be translated to superspace and that the superfield associated to the one loop order term of the action involves 
the anomalies and Wess Zumino terms. An interesting point that remains as an object of future investigation is the extension of this superspace formulation for the more general cases in which anomalies and Wess Zumino terms depend on the antifields.

\section{Acknowledgements}

The authors would like to thank Ashok Das for very important discussions. This work is supported in part by CNPq, FINEP, FAPERJ, FUJB and CAPES (Brazilian Research Agencies). 


\section{References}

[1] C. Becchi, A. Rouet and R. Stora, Comm. Math. Phys. 42 (1975) 127; I. V. Tyutin, Preprint Lebedev Inst. No. 39 (1975).

[2] Zinn-Justin,J. "Renormalization of gauge theories", in Trends in Elementary Particle Theory, Lecture Notes in Physics Vol. 37, ed. H.Rollnik and K.Dietz, Berlin, Springer (1974).

[3] L.D.Faddeev and V.N. Popov, Phys. Lett. B25 (1967) 29.

[4] I. A. Batalin and G. A. Vilkovisky, Phys. Lett. B102 (1981) 27, Phys. Rev. D28 (1983) 2567.

[5] M.Henneaux and C.Teitelboim, Quantization of Gauge Systems, Princeton University Press 1992, Princeton, New Jersey.

[6] J. Gomis, J. Paris and S. Samuel, Phys. Rep. 259 (1995) 1.

[7] W.Troost, P.van Nieuwenhuizen and A. Van Proeyen, Nucl. Phys. B333 (1990) 727.

[8] S. Ferrara, O. Piguet and M. Schweda, Nucl. Phys. B119 (1977) 493; K. Fujikawa, Progr. Theor. Phys. 59 (1978) 2045.

[9] N.R.F. Braga and A. Das , Nucl. Phys. B442 (1995) 655.

[10] J. Alfaro and P. H. Damgaard, Nucl. Phys. B404 (1993) 751.

[11] Superspace or One Thousand and One Lessons in Supersymmetry, S.J. Gates, M.T. Grisaru, M. Rocek and W. Siegel, 1983, Benjamin Cummings, Massachusetts. .

[12] M. Henneaux, "Lectures on the Antifield-BRST Formalism for gauge Theories", Nucl. Phys. B - Proc. Suppl. 18A (1990) 47.

[13] F. Brandt, Phys. Lett B320 (1994) 57.

[14] G. Barnich and M. Henneaux, Phys.Rev.Lett.72 (1994) 1588.

[15] J.Gomis and J.Paris, Nucl. Phys. B431 (1994) 378.

[16] J.Paris, Nucl. Phys. B450 (1995) 357.

[17] J.Gomis, K.Kamimura, J.M.Pons, and Z.Zamora, "One loop anomalies and Wess Zumino terms for general gauge theories", hep-th 9509012.

[18] W. Troost and A.Van Proeyen, Regularization and the BV formalism, presented at "Strings 93", Berkeley, May 1993. 
[19] F.De Jonghe, The Batalin-Vilkovisky Lagrangian Quantization scheme with applications to the study of anomalies in gauge theories, PH.D. thesis K.U. Leuven, hep-th 9403143.

[20] L. D. Faddeev, Phys. Lett. B145 (1984) 81; L. D. Faddeev and S. L. Shatashvili, Phys. Lett. B167 (1986) 225.

[21] N.R.F.Braga and H.Montani, Int. J. Mod. Phys. A8 (1993) 2569; J. Gomis, J. Paris Nucl. Phys. B395 (1993) 288.

[22] F.De Jonghe, R.Siebelink, W.Troost, Phys.Lett.B306(1993)295. 